\title{
Follow the ubiquitin
}

A new antibody to monoubiquitinated histone $\mathrm{H} 2 \mathrm{~B}$ ( $\mathrm{ubH} 2 \mathrm{~B}$ ) allows chromatin immunoprecipitation (ChIP) from mammalian cells to map ubiquitination patterns across the genome or to follow the modification of individual genes.

An elaborate bureaucracy of enzymes leaves instructions on the histones through posttranslational modifications such as phosphorylation, acetylation and methylation. One such modification, ubiquitination, has eluded detailed examination in mammalian cells. In yeast, mutations in the ubiquitin target sites have shown that mono-ubiquitination of histone H2B at Lys120 is an important signal for transcription initiation and elongation, and in the DNA-damage response. Mammalian cells, however, have $16 \mathrm{H} 2 \mathrm{~B}$ histone family genes, making such genetic studies difficult, and the lack of biochemical reagents precludes further studies. Now Moshe Oren and his colleagues at the Weizmann Institute of Science report the development of an antibody specific for histone H2B ubiquitinated on Lys120 in mammalian cells. This reagent enables ChIP studies to monitor histone $\mathrm{H} 2 \mathrm{~B}$ ubiquitination throughout the genome and in individual genes.

Oren is interested in understanding the molecular mechanisms of cancer initiation and progression, and in particular the activities of the p53 tumor suppressor. Among its other responsibilities, p53 recruits enzymes that modify histones to regulate gene expression. To investigate the role of $\mathrm{p} 53$, Oren says that they "wanted to perform [ChIP] to determine the extent of histone $\mathrm{H} 2 \mathrm{~B}$ ubiquitination at specific genomic sites, mainly those that are regulated by our friend p53." But this plan had a substantial obstacle: ubiquitinated target-specific antibodies have been difficult to generate. So Oren sought the advice of another Weizmann scientist, Matti Friedkin, who suggested and made a branched peptide of just the histone $\mathrm{H} 2 \mathrm{~B}$-ubiquitin conjugation site, including residues of both the histone $\mathrm{H} 2 \mathrm{~B}$ and the ubiquitin moiety, to focus antigen-recognition on this structure. Oren's graduate student Neri Minsky tested hundreds of the resulting antibodies before finding one that worked in ChIP experiments.

The researchers then found themselves on an excursion from their p53 studies to explore global histone ubiquitination. First, they examined the relationship between $\mathrm{ubH} 2 \mathrm{~B}$ and transcription by running their ChIP samples on microarrays to generate a high-resolution map of DNA sequences associated with this modification. The researchers discovered that histone $\mathrm{H} 2 \mathrm{~B}$ was ubiquitinated primarily at promoter-proximal regions of transcriptionally active genes. This pattern contrasted with that of another modification, histone $\mathrm{H} 3$ dimethylation at Lys4, which is associated with transcriptional activation and, in yeast, is dependent on histone $\mathrm{H} 2 \mathrm{~B}$ ubiquitination. The researchers detected $\mathrm{ubH} 2 \mathrm{~B}$ upstream and downstream of the transcription start site, but the level of dimethylation noticeably dipped at the promoter. From these differences in overlap, Oren says the histone codes "appear more absolute" whereas mammalian rules seem "more flexible."

Oren and colleagues next analyzed the $\mathrm{ubH} 2 \mathrm{~B}$ patterns at individual genes, returning to their original goal of analyzing histone $\mathrm{H} 2 \mathrm{~B}$ ubiquitination during the $\mathrm{p} 53$ response. They performed ChIP analysis of a prototypical target gene, CDKN1A (also known as p21), while manipulating $\mathrm{p} 53$ activity with a temperature-sensitive mutant of the tumor suppressor. The researchers detected $\mathrm{ubH} 2 \mathrm{~B}$ associated with the $p 21$ gene soon after p53 activation, but there was a rapid decline in $\mathrm{ubH} 2 \mathrm{~B}$ signal once $\mathrm{p} 53$ was deactivated; they found that histone $\mathrm{H} 2 \mathrm{~B}$ ubiquitination can have a half-life on the order of minutes.

Ultimately, Oren's team found this detour away from cancer biology profitable. They now better understand the role of histone $\mathrm{H} 2 \mathrm{~B}$ ubiquitination in mammalian cells and can finally follow ubH2B in p53 target genes with this valuable antibody. In fact, Oren feels "that the most important aspect of this study will be the introduction of this new tool, which will enable chromatin researchers to perform experiments that they may have wanted to do for a long time but couldn't. In other words, the questions are already there in a number of labs, drawn on the board, and all that is needed now is just to go ahead and do the next experiment."

\section{Katherine Stevens}

RESEARCH PAPER

Minsky, N. et al. Monoubiquitinated H2B is associated with the transcribed region of highly expressed genes in human cells. Nat. Cell Biol. 10, 483-488 (2008). 